\title{
Strom aus Licht - eine Wachstumsindustrie
}

\author{
F. Frisch ${ }^{1}$
}

\begin{abstract}
Die führenden Industrieländer verfolgen neuerdings ehrgeizige Ziele bei der Stromerzeugung aus Sonnenenergie. Die zweite Weltkonferenz für Photovoltaik in Wien machte erstmals deutlich, daß die umweltfreundliche Energiequelle das Potential hat, zu einem Global Player in der künftigen Weltenergieversorgung zu werden. Sie geht jetzt nach langer Pionierzeit in die industrielle Phase über. Ihr wirtschaftliches Wachstum wird bereits an der Computertechnik gemessen.
\end{abstract}

Schlüsselwörter: Photovoltaik; Industriephase; wirtschaftliche Anwendung; Weltenergieversorgung; Massenproduktion; Dünnschichttechnik

Generation of electric power from solar energy becomes growth industry. The major industrial nations have recently set ambitious goals for the generation of electric power from solar energy. The Second World Conference for Photovoltaics, held in Vienna, made it clear, for the first time, that this ecologically sound energy source has the potential to become a global player on the world energy scene. Following a long pioneering period, solar energy is now moving into the industrial phase. Its economic growth is already being compared with the growth of computer technology.

Keywords: photovoltaics; solar generators; industrial phase; economic applications; global energy supply; mass production; thin-film solar cells

\section{Einleitung}

In der Geschichte der Technik und der Industrialisierung haben sich immer wieder Erfindungen dynamischer durchgesetzt, als es Experten zunächst erwartet hatten. Dabei wurden oft erhebliche Widerstände überwunden. Auch die Photovoltaik, die direkte elektronische Umwandlung der Energie des Sonnenlichts in Elektrizität, wurde von ihren Anfängen in den sechziger Jahren bis in unsere Tage als zwar elegante, aber für die breite Stromversorgung unbedeutende Technik eingestuft, die allenfalls in der Raumfahrt und einigen terrestrischen Nischenmärkten eine Rolle spielen könne.

Diese Einschätzung beginnt sich jetzt zu wandeln. Die führenden Industrieländer verfolgen neuerdings ehrgeizige Ziele mit Stromerzeugung aus Sonnenenergie (Abb. 1). Die zweite Weltkonferenz für Photovoltaik in Wien machte erstmals deutlich, daß die umweltfreundliche Energiequelle das Potential besitzt, zu einem Global Player in der künftigen Weltenergieversorgung $\mathrm{zu}$ werden. Sie geht nach langer Pionierzeit in die industrielle Phase über. Ihr wirtschaftliches Wachstum wird

\footnotetext{
Dipl.-Ing. Franz Frisch, Publizist für Forschung und Technologie, Wertheimer Straße 93, D-81243 München.
}

bereits an der Computertechnik gemessen. Der weltgrößte Ölkonzern stellt sich auf eine massive Rolle der Photovoltaik bei der Ablöse des Erdöls ein. Nebenbei ist auch die erste Massenproduktion von Solargeneratoren für die Automobilindustrie angelaufen. Was sind die Gründe dafür, daß die elektronische Stromquelle plötzlich eine wirtschaftliche Eigendynamik entwikkelt?

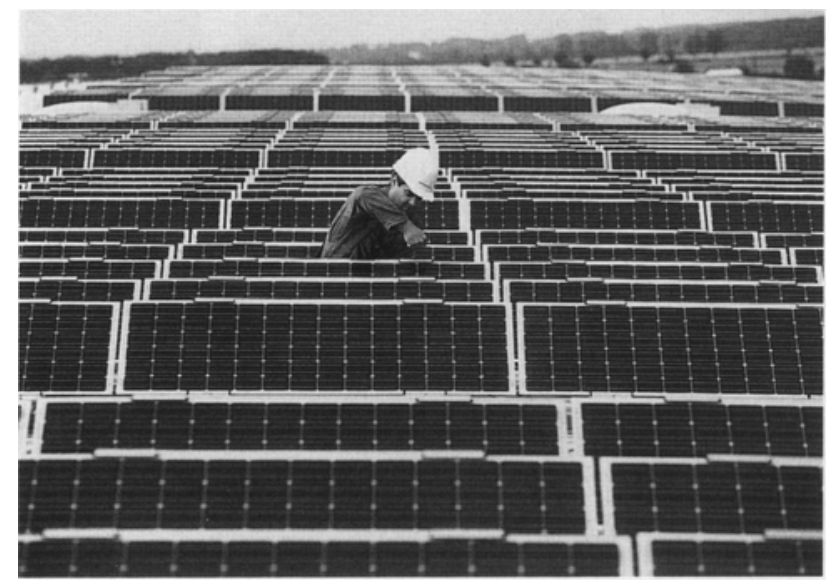

Abb. 1. Die erste deutsche Photovoltaik-Anlage mit einer Leistung von einem Megawatt auf den Dächern der Neuen Messe München soll die Marktentwicklung stimulieren

(Bild: Markus Matzel. Das Fotoarchiv) 


\section{Massive Programme zur Marktentwicklung}

Die Europäische Union will eine Million Minikraftwerke, die Sonnenenergie direkt in Strom umwandeln, bis zum Jahr 2010 installieren - die eine Hälfte in den Mitgliedsländern, die andere in der Dritten Welt. Die solare Stromkapazität soll damit auf $3000 \mathrm{MW}$ steigen. „Damit werden wir einen entscheidenden Marktdurchbruch der Photovoltaik erreichen", sagte Christos Papoutsis, der für Energie zuständige EU-Kommissar, auf der zweiten Weltkonferenz für Solarstrom, die im Juli in Wien stattfand.

In den USA rechne man für 2010 mit 60 Milliarden Dollar Marktvolumen, bekräftigte James E. Rannels vom amerikanischen Energieministerium (DOE). 1997 sei die PV-Produktion in den Vereinigten Staaten um $47 \%$ gestiegen (Abb. 2). Das starke Wachstum werde durch neue Märkte getragen und durch staatliche Initiativen wie das von Präsident Clinton initiierte Programm zur Installation von einer Million PhotovoltaikAnlagen auf amerikanischen Dächern.

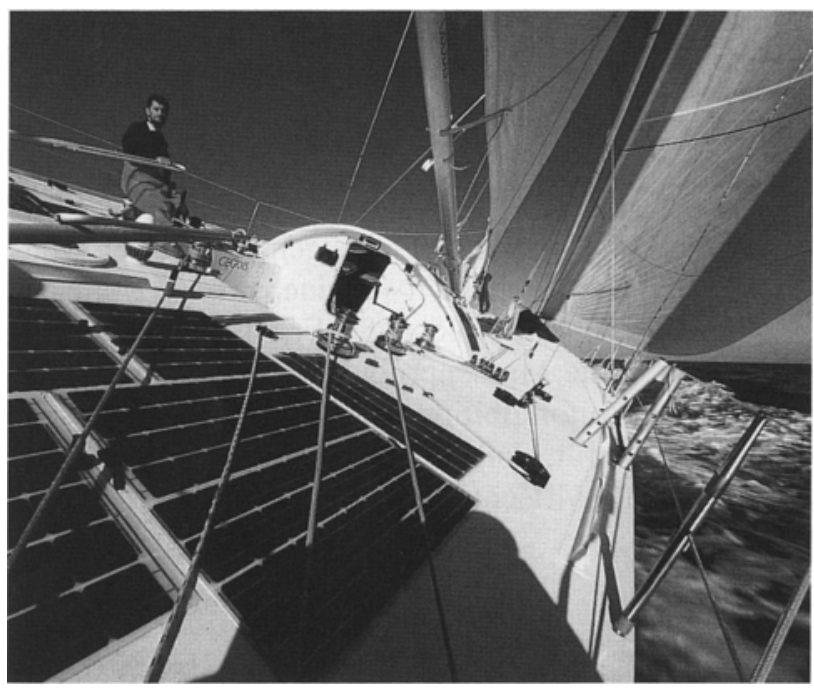

Abb. 2. Ein Markt von $60 \mathrm{Mrd}$. Dollar wird für 2010 in den USA erwartet. Nicht nur im Weltraum, sondern auch auf der Erde steigt die Nachfrage nach Elektrizität ohne Brennstoff und Abfall

(Bild: Webasto)

Die Statements der beiden Energiepolitiker charakterisieren den grundsätzlichen Wandel in der Einschätzung der solaren Stromerzeugung, der auf der Wiener Weltkonferenz erstmals deutlich zu Tage trat. Die Photovoltaik, die viele Vertreter von Politik und Wirtschaft bisher nur als Nischentechnik ohne Bedeutung für den Energiemarkt werteten, hat sich so dynamisch entwikkelt, daß sie nun als ernsthafter Wettbewerber im Weltenergiemarkt des 21. Jahrhunderts in Betracht gezogen wird.

\section{Parallele zur Computertechnik}

Über 2000 Experten und Anwender aus 75 Ländern nahmen an dem 5tägigen Treffen vom 6. bis 10. Juli in der Wiener Hofburg teil. 1100 Vorträge und Posterbeiträge zeigten den breiten Strom der Entwicklung. Bei der begleitenden, bisher größten Fachmesse der Photovoltaik präsentierten 150 Aussteller aus 21 Ländern ihre Produkte. Und hier zeigte sich bereits einer der Gründe für die neue Entwicklung: Wir haben es mit einer in der Anwendung bereits reifen Technik zu tun, die vielfältige Bedürnisse kostengünstig und qualitativ hochwertig deckt.

„Wir können heute zeigen, daß sich die Mikroelektronik, die inzwischen die technische Welt geprägt hat, in ihren ersten 15 Jahren nicht schneller entwickelte als die Photovoltaik", sagte der Vorsitzende der Wiener Weltkonferenz, Professor Jürgen Schmid, Vorstandsmitglied des Instituts für Solare Energieversorgungstechnik (ISET) in Kassel, in seiner Keynote-Rede. Die Photovoltaik durchlaufe zur Zeit jenes Stadium, in dem sich die Computertechnik in den 70er Jahren befunden habe. Sie sei wie die damaligen Computer technisch bereits hoch entwickelt und vielfältig einsetzbar. „Wir stehen aber gleichsam noch weit vor der Einführung des billigen PCs und dem Boom der Mikrochips.“

\section{Systementwicklung seit 15 Jahren}

Jürgen Schmid war einer der ersten, der in Europa systematisch an der Verbindung von Elektronik und Photovoltaik arbeitete. Bald nach der Gründung des bundesdeutschen Fraunhofer-Instituts für Solare Energiesysteme (ISE, Freiburg), heute das größte Solarinstitut Europas, baute er die ISE-Abteilung Systemtechnik auf, die sich fortan auf die vielfältige, maßgeschneiderte Anwendung der elektronischen Generatoren konzentrierte. Bereits 1983 nahm Schmid in München die erste europäische Wohnhausanlage in Betrieb, die aus Sonnenlicht mittels eines nahezu vollelektronischen Inverters exakten 220-V-Wechselstrom erzeugte und diesen bei Überschußproduktion sogar schon in das Münchner Stadtnetz einspeisen durfte. Wenig später folgte der erste Berggasthof ohne Netzanschluß, der durch eine Photovoltaikanlage mit Batteriespeicher solaren Wechselstrom rund um die Uhr erhielt.

Derartige Projekte, gefördert etwa von der Brüsseler EU-Kommission, haben entscheidend dazu beigetragen, von der Idee zur qualifizierten Anwendung zu kommen, die nun immer breiter wächst.

Ein modernes Beispiel stellt die Starkenberger Hütte des Deutschen Alpenvereins in Tirol dar. Hier hat das ISET Kassel mit modularer Systemtechnik einen PVGenerator mit 4,95 kW Spitzenleistung und ein mit Flüssiggas betriebenes Blockheizkraftwerk mit $14 \mathrm{~kW}$ Nennleistung zu einer Hybridanlage integriert, die die 


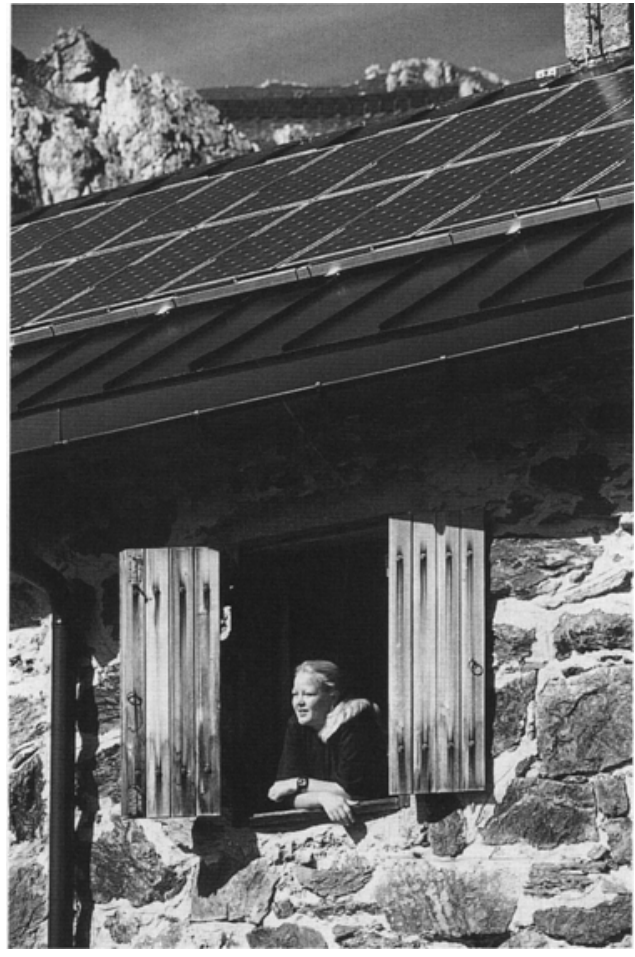

Abb. 3. Modulare Energieversorgung mit Photovoltaik und Flüssiggas in Tirol: In der Starkenberger Hütte des Deutschen Alpenvereins installierte das ISET Kassel ein maßgeschneidertes Stromsystem (Bild: Markus Matzel. Das Fotoarchiv)

Alpenhütte bei einem Minimum an Transportaufwand, Umweltbelastung und Wartung unter allen Witterungsbedingungen zuverlässig mit Strom und Wärme versorgt. Die Anlage wurde wegen ihres Pilotcharakters vom bundesdeutschen Forschungsministerium und der EU-Kommission gefördert (Abb. 3).

\section{Neue Perspektiven der Stromversorgung}

Zwei Gründe werden nach Schmids Worten dazu führen, daß die Photovoltaik ,wahrscheinlich zur stärksten Wachstumsbranche des nächsten Jahrhunderts wird." Der eine liegt darin, daß der Energieverbrauch der Menschheit stark steigen wird, Erdöl und auch Uran aber in wenigen Jahrzehnten zur Neige gehen und langfristig nicht mehr für die Weltenergieversorgung zur Verfügung stehen werden. Der andere Grund liegt in der einzigartigen Attraktivität der Photovoltaik:

„Zum ersten Mal“, so der Energieforscher, ,verfügt die Menschheit über eine Energiequelle ohne Grenzen des Wachstums.“ Die Ressourcen seien für alle Zeiten unbeschränkt verfügbar, eine Umweltbelastung wie bei konventionellen Energieträgern gebe es nicht.

„Erstmals kann an jedem beliebigen Punkt der Erde ohne Netzanschluß Strom erzeugt werden“, fügt Schmid hinzu. Begünstigt werde dies noch durch einen weiteren Vorteil: Die Größe der Generatoren lasse sich maßgenau an die Anwendungen anpassen, so daß nur die wirklich nötigen Kosten entstünden. Die Grundversorgung der gesamten Menschheit mit elektrischem Licht, Kühlung verderblicher Lebensmittel, Kommunikation mit TV und Telefon werde damit endlich realisierbar (Abb. 4). Eine Herausforderung für Technologieunternehmen in aller Welt sei schließlich, daß sich Photovoltaik als einzige Stromquelle direkt mit HighTech kombinieren lasse (Abb. 5).

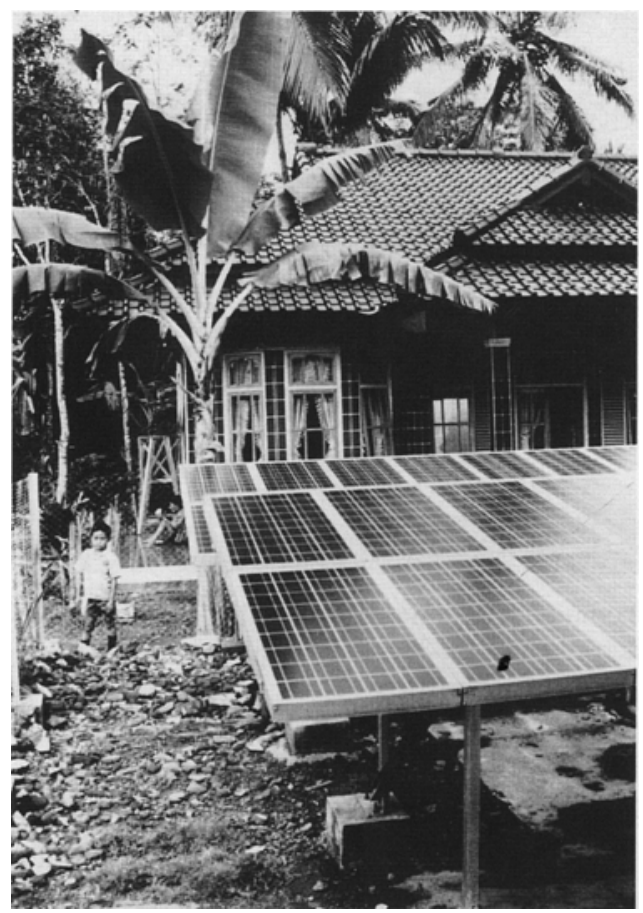

Abb. 4. Leistungsstarke Stromversorgung eines Wohnhauses in Indonesien: Mit Photovoltaik eröffnet sich ein praktikabler Weg, weltweit Versorgungslücken zu schließen

(Bild: ASE)

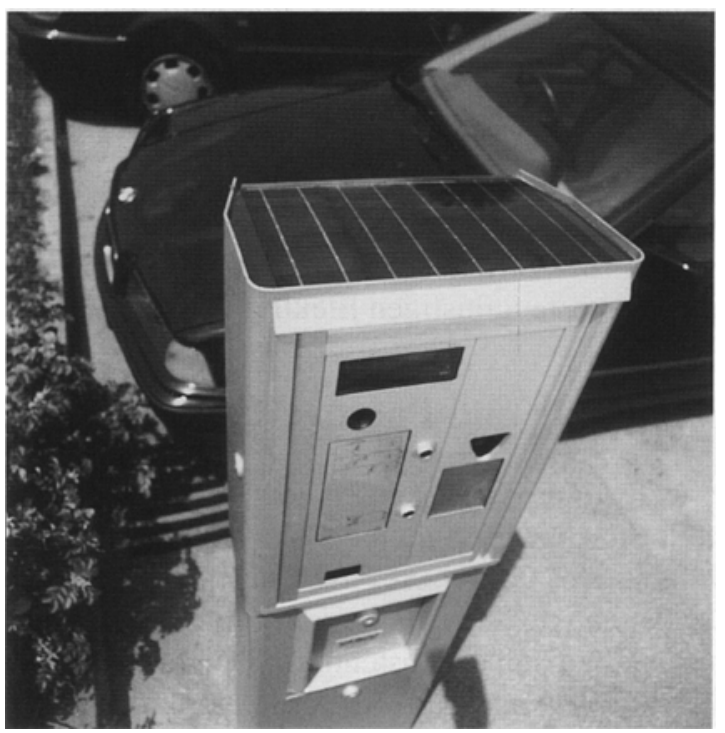

Abb. 5. Moderne Parkscheinautomaten mit photovoltaischer Energieversorgung sind bereits ein wirtschaftliches Serienprodukt, das auch mitten in deutschen Cities kostengünstiger ist als Geräte mit Netzanschluß (Bild: Webasto) 


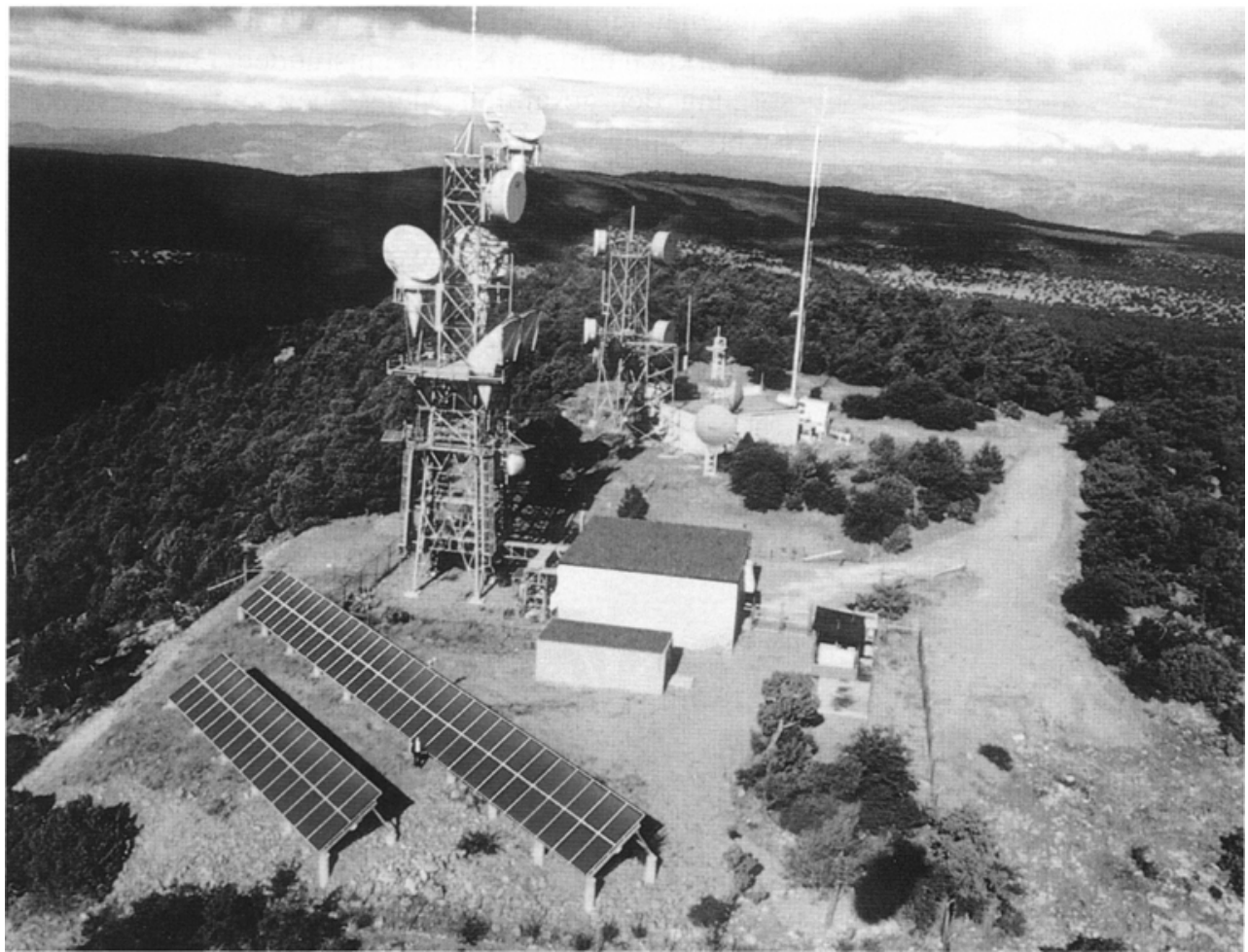

Abb. 6. Telekommunikation rund um den Globus wird durch Photovoltaik flächendeckend möglich. Hier hat sich ein Markt eröffnet, der das Wachstum des PV-Marktes forciert

(Bild: ASE)

Die Vorteile zeigen ihre Wirkung auf dem Weltmarkt. Das kontinuierliche Wachstum von jährlich über $20 \%$ wird bereits zu einem großen Teil durch Anwendungen vorangetrieben, bei denen die Solarstromversorgung die betriebswirtschaftlich günstigste Lösung darstellt. Die Liste der in Wien vorgestellten Projekte ist lang. Sie reicht von Telekommunikationsstationen rund um den Globus (Abb. 6) über Wasserpumpsysteme in Spanien, Holland und Deutschland bis zum höchsten Observatorium der Welt auf dem amerikanischen Mount Evans.

James E. Rannels betonte, es sei ein Irrtum, zu glauben, der Markt der Photovoltaik liege nur in den dünn besiedelten Entwicklungsländern. Auch in den hochentwickelten Industrieländern gebe es viele weiße Stellen in der kostengünstigen Elektrizitätsversorgung.

\section{Architektur und Photovoltaik}

Als ein weltweiter Trend der Photovoltaikanwendung entwickelt sich die Verbindung von Architektur und Photovoltaik. Der Grund: Stromerzeugende Fassadenoder Dachelemente ersetzen herkömmliche Baustrukturen und fallen daher kostenmäßig nicht stark ins Gewicht. Flächen sind im Übermaß vorhanden, der erzeugte Strom kann im Gebäude direkt verbraucht werden. Die beiden internationalen Glaskonzerne Saint Gobain und Pilkington sowie andere Firmen zeigten in Wien, daß sie diese Variante massiv voran- treiben wollen. Mit gutem Grund: „40\% der Elektrizität werden in Europa in Gebäuden verbraucht", sagt EU-Kommissar Papoutsis.

Eine reizvolle Alternative für Fassaden oder Dachflächen stellen großflächige semi-transparente Solarmodule in Dünnschichttechnik dar. Die Generatoren aus amorphem Silicium der ASE-Gruppe (PST, Putzbrunn bei München) lassen ca. $10 \%$ des Lichts durch. Dem menschlichen Auge erscheinen sie aus dem Gebäudeinneren transparent, von außen jedoch als undurchsichtige verspiegelte Fläche (Abb. 7).

\section{Firmen im Wettbewerb}

Photovoltaik-Firmen liefern ihre Produkte in alle Erdteile, so der vor allem in den USA produzierende heutige Weltmarktführer Siemens Solar, die amerikanische Solarex, die japanische Kyocera oder die deutsch-amerikanische ASE Gruppe, welche die zur Zeit fortschrittlichste Technologie zur Herstellung kristalliner Silicium-Wafer für Solarzellen nach dem EFG-Verfahren besitzt: Die hauchdünnen Scheiben werden nicht mehr wie Computersilicium mit viel Materialverlust aus großen Kristallblöcken gesägt, sondern wachsen in Gebrauchsdicke als achteckiges Rohr aus der Siliciumschmelze. Entlang der Kanten teilt ein feiner Laserstrahl das Rohr in quadratische Stücke (Abb. 8). 


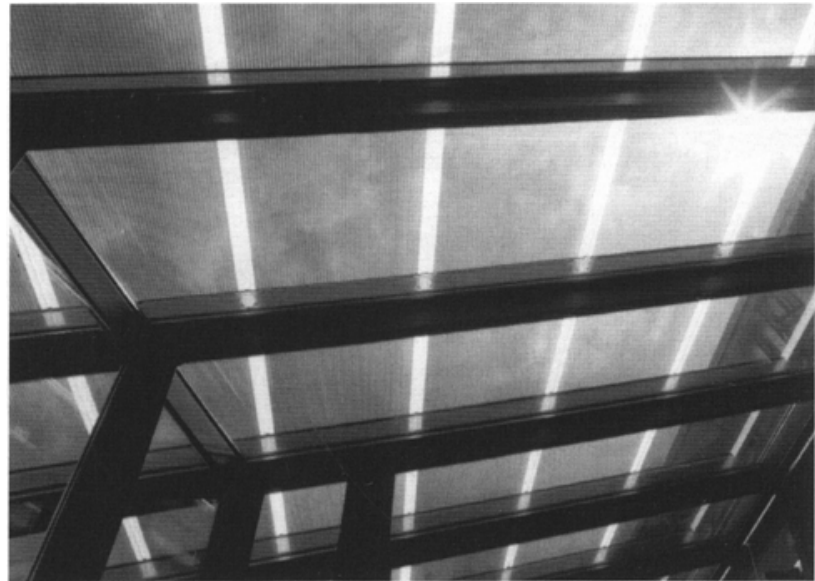

Abb. 7. Architektur und Photovoltaik: Semitransparente Module mit amorphem Silicium, hier in der Baubehörde von München, bieten neue Perspektiven bei der Gestaltung von Gebäuden (Bild: Phototronics Solartechnik)

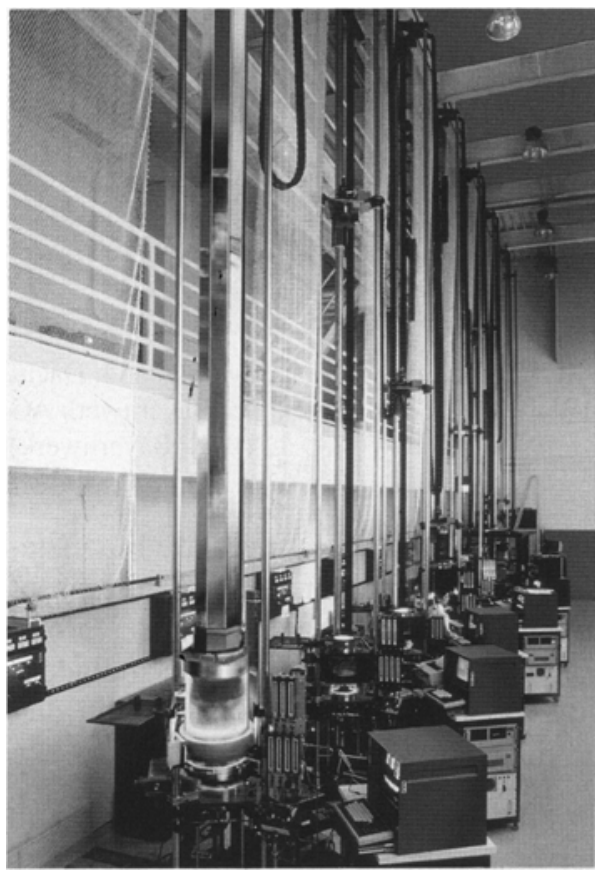

Abb. 8. Waferproduktion in Billerica, USA: Bei der fortschrittlichen EFG-Technologie entstehen von vornherein dünne Siliciumscheiben

(Bild: ASE)

„Elektrizität, unabhängig von Leitungen, wird in den kommenden Jahren völlig neue Anwendungsfelder und Marktsegmente erschließen", sagt Geschäftsführer Dr. Winfried Hoffmann. Die ASE GmbH (Angewandte Solarenergie), die heute zu $100 \%$ der Lahmeyer AG des RWE-Konzerns gehört, will ihren Weltmarktanteil (heute $5 \%$ ) bis zum Jahr 2000 verdoppeln und hat dazu vor kurzem im deutschen Alzenau eine neue Solarzellenfabrik mit 13 MW Jahresausstoß in Betrieb genommen. Sie wird damit ab 1999 in den USA und Deutschland Siliciumwafer und kristalline Solarzellen mit insgesamt $18 \mathrm{MW}$ Jahreskapazität produzieren.

\section{Gigawatts kommen in Sicht}

Zur Zeit kostet PV-Strom aus Großanlagen in Deutschland nach den Worten Hoffmanns noch 1,40 bis 1,50 DM pro Kilowattstunde. In sonnenreichen Ländern, etwa in Griechenland oder im Süden der USA sinke der Preis durch die höhere Sonneneinstrahlung auf die Hälfte. „Wir halten es für realistisch, daß bis 2010 dieser Preis halbiert werden kann. Das wären dann 20 Cents in den USA." Bei dieser Marke sei die Photovoltaik für die Deckung der Spitzenlast in der Stromversorgung interessant. Dann werde man etwa in Kalifornien beginnen, viele Gigawatt Photovoltaik zu installieren.

Hier liegt wohl ein ganz entscheidender Vorteil, den die Photovoltaik schon heute gegenüber der Kernkraft besitzt: Die Herstellung von Solarstromanlagen wird heute für Unternehmen kalkulierbar. Es werden Märkte sichtbar, die Investitionen als lohnend erscheinen lassen. Die Risiken sind überschaubar. Demgegenüber wird Kernenergie für private Unternehmen wirtschaftlich nur dann beherrschbar sein, wenn erhebliche Kosten und Risiken auf Staat und Gesellschaft abgewälzt werden. Bei der Zukunftsoption der Kernfusion dürfte dies nicht anders sein.

Die erste Ein-Megawatt-Anlage in Deutschland, von Bayernwerk, Siemens und den Stadtwerken München auf den Hallendächern der neuen Münchner Messe errichtet, demonstriert eine weitere PV-Entwicklung: Mit vielfältigen großen Demonstrationsanlagen, die Solarstrom ins öffentliche Netz einspeisen, soll die Marktentwicklung stimuliert werden (Abb. 9). Eine zweite Anlage mit einem Megawatt Leistung entsteht zur Zeit auch auf dem riesigen Dach der neuen Bildungsakademie Herne in Nordrhein-Westfalen. Mit ähnlichem Ziel will auch die japanische Regierung 70000 Dächer mit PV-Anlagen ausstatten.

\section{Zwei Milliarden Menschen ohne Strom}

Eine der wichtigsten Herausforderungen der Photovoltaik stellen die über zwei Milliarden Menschen dar, die heute nach wie vor ohne Elektrizität leben. Dazu zählen auch eine Million EU-Bürger. Ihnen eröffnet die elektronische Energietechnik, die ohne Netzanschluß, Brennstoff und Abfall Strom erzeugt, eine neue Lebensperspektive. So wurde in Wien über viele Beispiele berichtet, die zeigen, wie die Elektrifizierung der dünnbesiedelten Gebiete der Erde vorangehen kann: Elektrisches Licht für 1000 Häuser in Ladakh, Wasserpumpen in Senegal und Mexico, Elektrifizierung in Indien und Cuba usw. Der entscheidende erste Schritt ist die elektrische Basisversorgung, die für TV, Licht, Kühlschrank und ein weiteres Elektrogerät reicht. Im vergangenen Jahr wurden nach einer Analyse des Freiburger Fraunhofer-Solarinstituts ISE weltweit bereits 200000 solche 


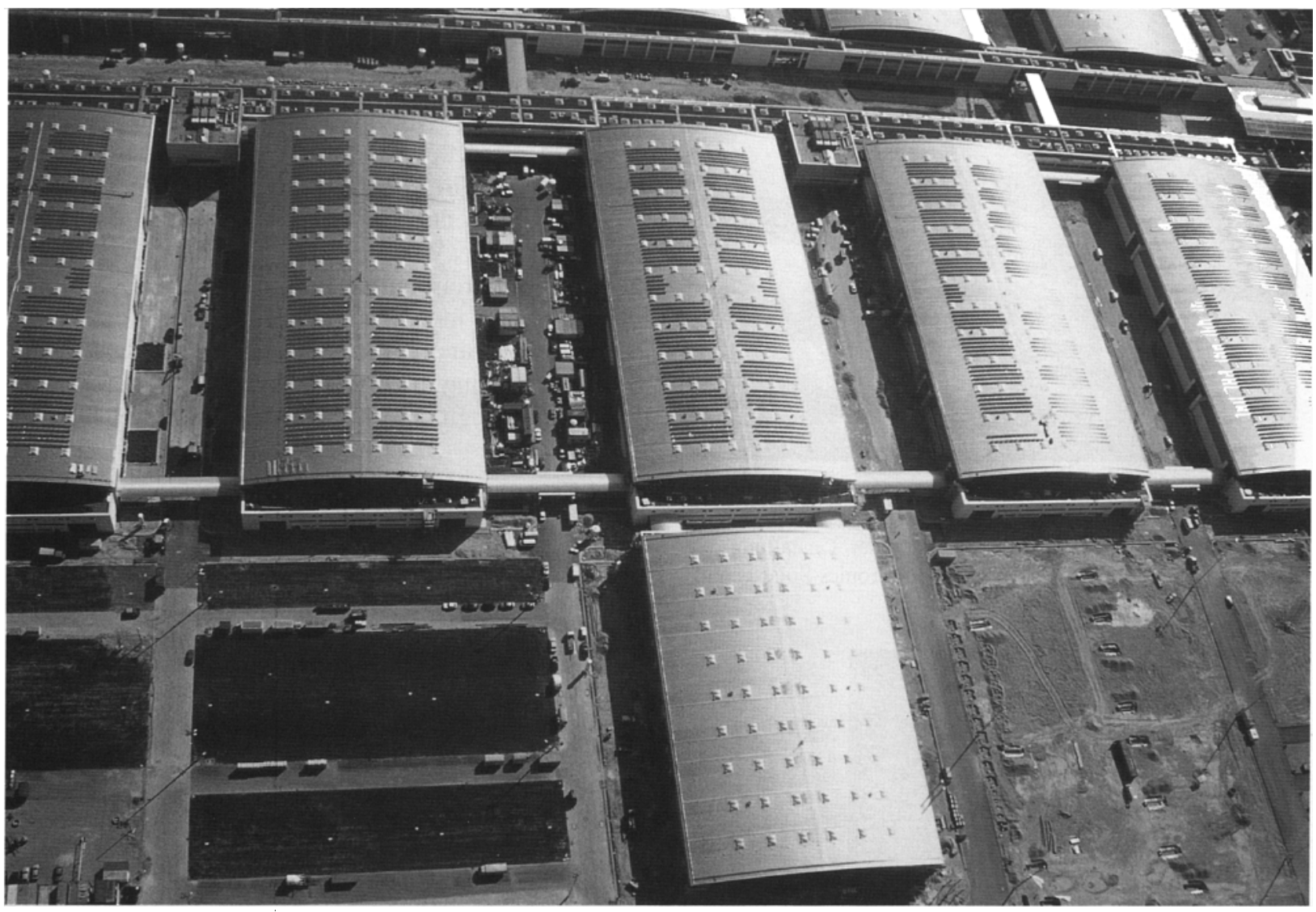

Abb. 9. Demonstrationslage zur Marktförderung auf den Hallendächern der Neuen Messe München: Auf einer verfügbaren Dachfläche von $66000 \mathrm{~m}^{2}$ wurden 7812 Solarmodule mit einer Gesamtleistung von $1016 \mathrm{MW}$ installiert. Ein Projekt der Bayernwerk AG (Hauptinvestor), der Siemens AG und der Münchner Stadtwerke

(Bild: Bayernwerk)

Solar-Home-Systems installiert, die den Menschen neue Bildungs- und Arbeitsmöglichkeiten bieten und damit zur Reduzierung der Landflucht beitragen (Abb. 10).

\section{Größter Ölkonzern steigt massiv ein}

Die Nachfrage ist groß und auch das Kapital für die Investitionen wäre vorhanden, war in Wien zu hören.

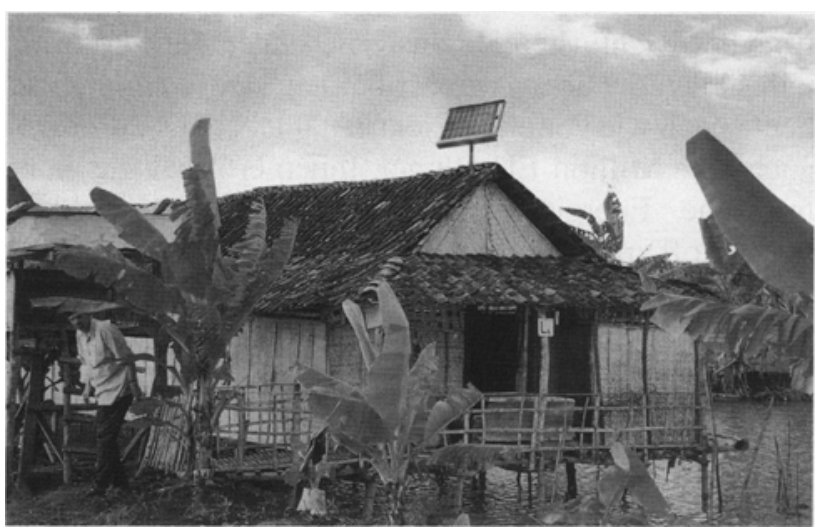

Abb. 10. Über zwei Milliarden Menschen leben noch ohne Elektrizität. Mit photovoltaischen Solar-Home-Systems, hier in Indonesien, kann ihre Versorgung eingeleitet werden

(Bild: ASE)
Was fehlt, sind geeignete, maßgeschneiderte Finanzierungsmodelle der Kreditwirtschaft. Auch dazu präsentierte der Weltkongreß erste Konzepte. Als Vorbild wurde vor allem die Gramehn-Bank in Bangladesh genannt, die mit großem Erfolg Kleinprojekte in diesem armen Land finanziert.

Die globalen Zukunftsperspektiven der Photovoltaik sind heute so vielversprechend, daß der Shell-Konzern, das größte Ölunternehmen der Welt, regenerative Energie im Herbst 1997 zu seinem fünften Geschäftsfeld erhoben hat. „Nach dem offziellen Energieszenario von Shell wird der Anteil der Photovoltaik am gesamten Weltenergieverbrauch im Jahr 2050 bei $10 \%$ liegen", sagt Dr. Fritz Vahrenholt, Vorstandsmitglied der deutschen Shell AG. „Der Weltenergieverbrauch wird zu diesem Zeitpunkt dreimal so hoch sein wie heute." Entscheidend bei der langfristigen Entwicklung der Photovoltaik sei nicht das Anfangsvolumen des Marktes, sondern der Gradient des Wachstums. Shell halte ein jährliches Wachstum bis zu $30 \%$ für realistisch. Damit könne bis zum Jahr 2020 ein Prozent Marktanteil erreicht werden. Zwischen 2030 und 2050 könne dann der Anteil von $5 \%$ auf $10 \%$ steigen. Diese Entwickung sei jedoch „kein Selbstläufer“. Es seien noch große Anstrengungen und viele technologische 
Quantensprünge nötig, um das Ziel zu erreichen. Shell errichtet zur Zeit in Gelsenkirchen die weltgrößte Photovoltaik-Fabrik mit einer Jahreskapazität von $25 \mathrm{MW}$ (Abb. 11).

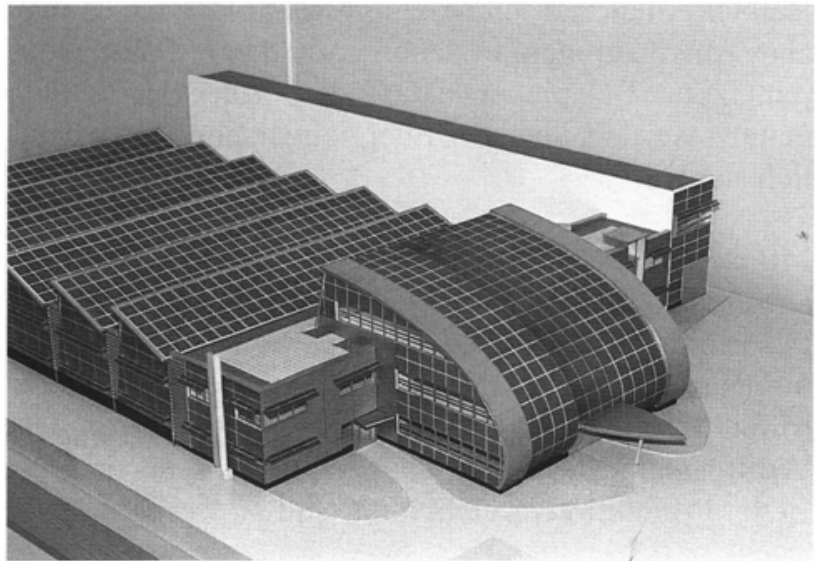

Abb. 11. Modell der weltgrößten Solarzellenfabrik, die von Shell im deutschen Gelsenkirchen errichtet wird

(Bild: MFF)

\section{Erste Massenproduktion für das Auto}

$\mathrm{Zu}$ den wichtigsten Unternehmen, die den Weltmarkt der Photovoltaik erweitern, werden künftig aber auch viele High-Tech-Unternehmen zählen, die mit speziellen Produkten auf ihrem Markt führend sind. Ein Paradebeispiel gibt im Automobilbereich die Firma Webasto, Weltmarktführer bei Schiebedächern, die ihre Produkte heute an praktisch alle Autohersteller rund um den Globus liefert. ,Wir beschäftigen uns täglich mit unserem Kundennutzen Licht, Luft, Sonne“, sagt Reinhard Wecker, Leiter der Webasto-Solartechnik. „Was lag näher, als das Schiebedach mit Solarzellen auszurüsten, um auch im stehenden Fahrzeug eine gute Belüftung zu erreichen." Die bayerische Weltfirma ist damit heimlich zu einem großen deutschen Solartechnik-Hersteller geworden. In diesem Jahr wird man 20000 bis 30000 Solar-Schiebedächer an die Autoindustrie und andere Branchen liefern. Im Spitzenmodell von Audi, dem A8, gehört das Solardach bereits zum Klimapaket, das als Sonderausstattung angeboten wird (Abb. 12).

\section{Neue Technologien gehen in Produktion}

Der Weg zum Global Player in der Weltenergieversorgung führt über die Massenproduktion neuer Halbleitertechnologien, die die Stromerzeugungskosten senken. Zukunftstechnologien, die den Materialaufwand für die Solarzellen drastisch reduzieren, nahmen in Wien denn auch einen breiten Raum ein. Dünnschichtzellen, mehr als hundertmal dünner als die heutigen kristallinen Zel-

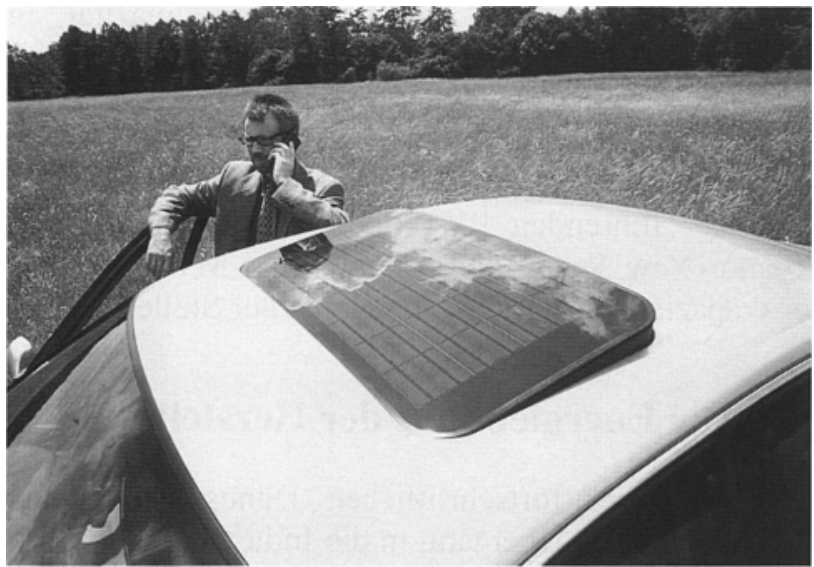

Abb. 12. Erste Massenproduktion für die Automobilindustrie: Das Schiebedach mit Solargenerator, hier im Audi A8, erfüllt die hohen Qualitätsanforderungen der Pkw-Hersteller

(Bild: Markus Matzel. Das Fotoarchiv)

len, sind diesen im Wirkungsgrad noch unterlegen. Doch bei vielen Anwendungen kommt es nicht auf höchste Effizienz an, sondern auf die Stromkosten pro Watt, und hier bieten Dünnschichtzellen auf Grund ihrer sinkenden Produktionskosten die besten Perspektiven.

Die älteste Variante, das amorphe Silicium, das in den achtziger Jahren fast begraben worden wäre, erlebt einen neuen Aufschwung. Erstmals, so berichtete die amerikanisch-japanische Firma Uni-Solar, seien im Labor $15 \%$ Wirkungsgrad überschritten worden. Zum Vergleich: der Laborwirkungsgrad kristalliner Hochleistungszellen liegt bei $24 \%$. Nach bisheriger Erfahrung sind in der Produktion etwa zwei Drittel des höchsten Laborwerts wirtschaftlich zu erreichen.

Zur Zeit liegt der stabile Wirkungsgrad amorpher Siliciumzellen noch bei $6 \%$ bis $6,5 \%$. Großflächige Module produziert ASE bereits seit längerem in einer

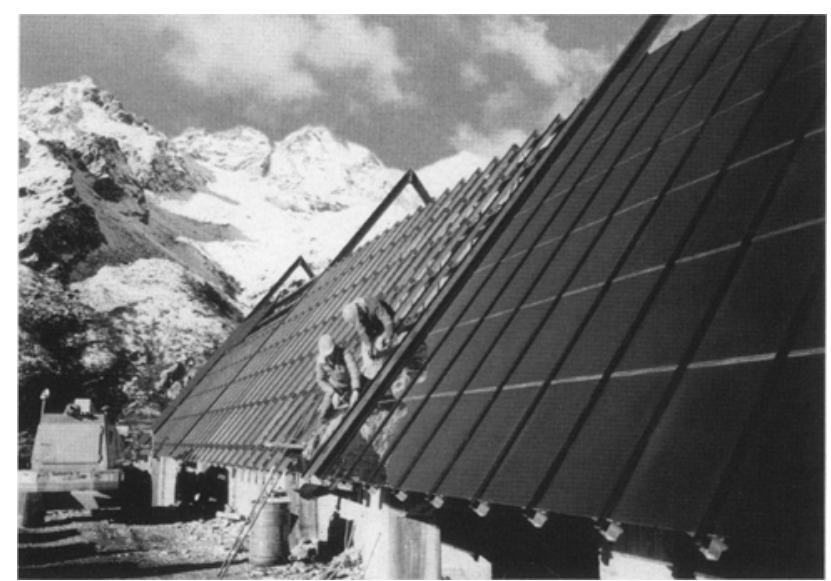

Abb. 13. Zukunftsträchtige Dünnschichttechnologie: Hauchdünne Schichten aus amorphem Silicium liefern am Telessio Damm in Italien Strom mit $12 \mathrm{~kW}$ Leistung

(Bild: ASE) 
Pilotfabrik mit einem Megawatt Jahreskapazität. In den nächsten zwei bis drei Jahren stehe, so das Unternehmen, die Entscheidung für eine Ausweitung der Produktion auf $10 \mathrm{MW}$ bis $15 \mathrm{MW}$ an (Abb. 13). Die größte Produktion mit $10 \mathrm{MW}$ hat zur Zeit Solarex, eine der führenden PV-Firmen, im amerikanischen Toano (New Jersey) aufgebaut. Mit 5 MW Produktionskapazität steht Uni-Solar an zweiter Stelle.

\section{Die Energiebilanz der Herstellung}

Auch bei der fortschrittlichen Dünnschichttechnik wird bereits der Übergang in die Industriephase sichtbar. Obwohl die Wirkungsgrade noch verhältnismäßig klein sind, starten Firmen große Produktionen, um gerüstet zu sein, wenn das jetzt schon sichtbare Kostensenkungspotential in der Zukunft auf dem Markt umgesetzt wird.

Die Grundbedingung für eine massive Rolle der Photovoltaik in der Weltenergieversorgung ist ein möglichst kurzfristiges Energy-Pay-Back - die elektronischen Generatoren müssen die Energiemenge, die zu ihrer Herstellung eingesetzt wurde, durch Solarenergieumwandlung möglichst schnell wieder hereinbringen. In einem gemeinsamen Bericht haben Forscher der Universität Utrecht, des französischen INSEAD-Instituts und der japanischen AIST-Forschungsgesellschaft den aktuellen Stand dargelegt. Danach beträgt die EnergyPay-Back-Time (EPBT) einer kompletten PV-Anlage mit $3 \mathrm{KW}$ Leistung bei Verwendung von amorphen Siliciumzellen nur mehr 1,1 Jahre, wenn pro Jahr derartige Anlagen mit einer Gesamtleistung von $100 \mathrm{MW}$ hergestellt werden. Schon heute garantieren Hersteller 10 Jahre Lebensdauer der Solarmodule. Die tatsächliche Funktionszeit dürfte nach bisherigen Erfahrungen
20 und mehr Jahre erreichen, so daß bei heutiger Technologie Dünnschichtzellen bereits $20 \mathrm{mal}$ so viel Strom gewinnen können, wie sie bei der Produktion verbrauchen. Diese Rechnung umfaßt jedoch alle Komponenten der Anlage, u. a. auch die Glasplatte, auf die das Halbleitermaterial aufgedampft wird. Betrachtet man aber Anwendungen, in denen vorhandene Baustrukturen, zum Beispiel Glasfassaden von Hochbauten genutzt werden, kann der Energieüberschuß noch deutlich steigen.

Mit dem ersten Produktionsstart der zweiten Dünnschichttechnologie, Solarzellen aus Kupfer-IndiumDiselenid (CIS), machte Siemens Solar auf sich aufmerksam. Für den Anfang will man in den USA kleine Module mit $5 \mathrm{~W}$ und $10 \mathrm{~W}$ Leistung produzieren. Der Wirkungsgrad liegt im Labor inzwischen bei $17,7 \%$. In der Produktion erreicht man rund $10 \%$.

In Produktion geht auch die dritte Dünnschichttechnik, die eine Verbilligung in Aussicht stellt: Solarzellen aus Cadmiumtellurid. Ihr Wirkungsgrad liegt, zwischen amorphem Silicium und CIS, bei $8 \%$ bis $10 \%$. Zur Zeit sind zwei Fabriken mit jeweils $10 \mathrm{MW}$ Jahreskapazität im Bau, die eine wird vom Ölkonzern BP einer der heute führenden Photovoltaik-Firmen - in Fairfield, Kalifornien, errichtet, die andere von der neu gegründeten Firma Antec Solar im deutschen Rudisleben bei Erfurt.

Die nächsten Technologiegenerationen werfen aber ihre Schatten schon voraus. So präsentierten Forscher der Universität von Neuchâtel sogenannte mikrokristalline und mikromorphe Solarzellen. Und ein japanisches Team brachte die Nanotechnologie ins Spiel, die wiederum eine eigene Welt neuer Werkstoffe eröffnet. 